\title{
Análisis de la Percepción del Medio Ambiente de los Estudiantes de Ingeniería en Mecatrónica
}

\author{
Piero Espino-Román, Eugenia Olaguez-Torres e Yasser A. Davizon-Castillo \\ Universidad Politécnica de Sinaloa, Depto. Ingeniería Mecatrónica, Carretera Municipal Libre Mazatlán \\ Higueras Km3, Col. Genaro Estrada, C.P. 82199 Mazatlán, Sinaloa-México. \\ (e-mail: pespino@upsin.edu.mx; jolaguez@upsin.edu.mx; ydavizon@upsin.edu.mx)
}

Recibido Dic. 3, 2014; Aceptado Ene. 20, 2015; Versión final recibida Mar. 10, 2015

\begin{abstract}
Resumen
Se presenta un estudio acerca de la percepción de lo que es el medio ambiente y el nivel de conocimiento sobre aspectos ambientales en los estudiantes de la carrera de Ingeniería Mecatrónica de la Universidad Politécnica de Sinaloa, México. Para ello se ha trabajado en la elaboración de un instrumento de recopilación de datos, el cual consiste en la aplicación de un cuestionario integrado por 31 preguntas de escala tipo Likert, aplicado a 116 estudiantes. El análisis de contenido de las respuestas ha permitido analizar las actitudes y valores en los estudiantes con respecto a ecología y medio ambiente y la relación del ambiente con la ciencia y tecnología. Se concluye que se debe incluir la materia de educación ambiental en los planes de estudio de la carrera de Ingeniería Mecatrónica.
\end{abstract}

\section{Analysis about Perception of the Environment of Students in a Mechatronics Engineering Program}

\begin{abstract}
A study on the perception of what is the environment and what is the level of knowledge about environmental aspects in the students of Mechatronics Engineering at the Polytechnic University of Sinaloa, Mexico is presented. For this, an instrument of data gathering was constructed, which consisted of a questionnaire including 31 questions of Likert-type scale, applied to 116 students. The analysis of content of the responses allowed analyzing the attitudes and values in students with regard to ecology and environment and the relationship of the environment to science and technology. It is concluded that a course on environmental education must be included in the program of Mechatronics Engineering.
\end{abstract}




\section{INTRODUCCIÓN}

La sociedad contemporánea se enfrenta a nuevos retos relacionados con la gestión del ambiente. Actualmente se sufren las consecuencias del proceso de industrialización acaecido en las últimas décadas. La Unión Mexicana de Asociación de Ingenieros señala que el fenómeno del calentamiento global, ha puesto sobre la mesa de discusión, la necesidad de analizar y evaluar el impacto que tienen los patrones de producción y consumo de energía, debido a la importancia que tienen los recursos fósiles en la oferta total de energía primaria en el mundo. A nivel mundial, casi el $70 \%$ del total de emisiones de gases de efecto invernadero (GEI) tienen que ver con usos energéticos, de los cuales, $24 \%$ corresponden a la generación de energía, $14 \%$ al sector industrial, $14 \%$ al sector transporte, $8 \%$ al sector residencial y comercial, y el restante a otros usos relacionados con la energía (UMAI, 2009). Además, la deforestación de grandes extensiones con lo que el problema se agudiza al disminuir una fuente natural de captura del bióxido de carbono. Resulta evidente que el cambio climático, la pérdida de la biodiversidad, el deterioro ambiental, las emergencias producto de los desastres naturales, la escasez de agua, entre otros problemas, constituyen una realidad que está modificando los patrones de vida actuales y que compromete las expectativas de las generaciones futuras (Nelleman et al, 2010). Además de considerar las condiciones de pobreza, marginación y exclusión social, así como el agotamiento de la base de recursos naturales, altos niveles de contaminación ambiental y graves procesos de degradación ecológica (Márquez et al, 2011). Rodríguez (2007), señala que la ingeniería ha contribuido a generar muchos de esos cambios en la naturaleza, entre los cuales se encuentran aquellos de gran beneficio para la población, pero también aquellos que se consideran insostenibles desde la perspectiva ambiental y social. Por lo que los conocimientos y recursos en materia de ciencia, ingeniería y tecnología deben utilizarse para satisfacer las necesidades humanas básicas, reducir la pobreza, lograr el desarrollo sustentable (CAETS, 2009). Echeverría (2011) plantea que el futuro de la ingeniería depende de la respuesta fáctica que seamos capaces de dar como sociedad a un reto fundamental: el integrar de manera sistémica y ecológica la actividad ingenieril con las demás actividades sociales creadoras de valor.

Un informe de la Organización de las Naciones Unidas para la Educación, la Ciencia y la Cultura (UNESCO, 2006), señala que actualmente las sociedades del conocimiento están cada vez más internacionalizadas, en las que la ciencia, la ingeniería y la tecnología revisten una creciente importancia para el desarrollo social y económico. La Ingeniería Mecatrónica, en su actuación profesional, desarrolla las competencias para diseñar, mantener y automatizar dispositivos y sistemas, a través de la integración de conocimientos y tecnologías de la mecánica, electrónica, eléctrica, control y sistemas computacionales. La cual contribuye a la transformación económica, social y ambiental de nuestra época. Es evidente la necesidad de contribuir desde la Ingeniería Mecatrónica para establecer políticas, estrategias de desarrollo sustentable y metodologías de evaluación de indicadores de sustentabilidad (Espino et al, 2011). Ya que los futuros profesionales de Ingeniería Mecatrónica son responsables indirectos sobre las actuaciones que se realizan sobre el medio ambiente; pues no sólo deben incorporar los aspectos ambientales a la hora de diseñar y llevar a cabo los proyectos, sino también deben desarrollar la suficiente sensibilidad ambiental y social para poder incorporarlas a la ética en el trabajo. De ahí que sea oportuno cuestionar: ¿Cómo evaluar la percepción del medio ambiente de los estudiantes de ingeniería Mecatrónica?, ante el deterioro ambiental y el agotamiento de los recursos naturales que impactan persistentemente en la calidad de vida de las personas. Con el objeto de abordar las necesidades planteadas anteriormente; en el presente trabajo se pretende evaluar el conocimiento y compromiso ambiental en los estudiantes de la carrera de Ingeniería Mecatrónica de la Universidad Politécnica de Sinaloa, México; definir si éstos cuentan con la suficiente educación ambiental para contribuir en la lucha por preservar el medio ambiente específicamente al desarrollar una conciencia ambiental que motive una conducta comprometida con la preservación del medio ambiente.

La preocupación social por el ambiente, permitió que diversos estudios desarrollen instrumentos de medición respecto a las actitudes ambientales. Cerrillo (2010) hace referencia al trabajo formulado por Riley E. Dunlap en el que consolida el concepto de conciencia ambiental y de esta manera, entender el grado de preocupación de las personas por los problemas ambientales, el interés de apoyar iniciativas para solucionarlos, así también indicar la voluntad de participar con acciones concretas. En el estudio realizado por Zamorano et al. (2011) explica que los estudiantes tienen la capacidad de identificar la problemática ambiental actual, así también comprenden los daños que ocasiona la contaminación en general, el estudio se aplicó para estudiantes de educación media superior. Castells et al. (2012), profundiza la percepción del medio ambiente y los problemas mundiales de los estudiantes españoles, a partir de preguntas abiertas de cuestionarios, la investigación analiza la opinión y actitudes de 1267 estudiantes de centros de educación secundaria. Espejel et al. (2012) aborda aspectos de modelo de educación ambiental y su relación con programas ambientales, las actividades ambientales, la investigación se desarrolla en el marco de la metodología cualitativa, donde se aplicó 26 entrevistas divididas en tres apartados: a) actividades realizadas, b) organización de equipos y c) satisfacción de realizar acciones ambientales. La investigación 
presentada por Aragonés et al. (2006) tiene como objetivo analizar el concepto de problema ambiental que las personas perciben, atendiendo a la sensibilidad que diversas variables sociodemográficas tales como: edad, género, ideología política e intención de conducta proambiental tienen sobre la percepción de los problemas ambientales. Sosa et al. (2010), analizan la situación que guarda la educación ambiental en el nivel superior, el estudio consistió en la utilización de métodos cuantitativos y cualitativos para diagnosticar el grado de cultura ambiental en los estudiantes de la Universidad Autónoma de Campeche, los resultados del estudio muestran un bajo nivel de cultura ambiental, señala que el contexto institucional opera como un factor que inhibe la educación ambiental debido a que la formación ambiental no es considerada prioritaria y por lo tanto no existen los espacios, la infraestructura y los apoyos necesarios para su estudio, enseñanza y promoción. El vínculo entre las actitudes ambientales de los individuos y la concepción de la naturaleza y medio ambiente es presentado por Padilla et al. (2009), quienes estudian la relación existente entre algunos elementos microsociales en el espacio económico del turismo, se detectó la existencia de percepciones diferentes según los patrones de interacción social de grupos de individuos, dados por el carácter de su actividad práctica socioproductiva, o de los servicios que realizan, e incluso por su nivel educacional. En la investigación presentada por Márquez et al. (2011) indica que los estudiantes poseen un nivel de cultura ambiental bajo y carecen de conocimientos y habilidades necesarias para realizar cambios ambientales favorables en sus estilos de vida, el estudio permitió identificar actitudes positivas en los estudiantes para aprender prácticas sustentables así como la importancia que tiene la escuela como fuente de información ambiental. La percepción ambiental debe ser un soporte para incrementar la conveniencia, viabilidad y funcionalidad de la planificación ambiental. Barraza at al. (2003) señalan que la percepción ambiental se convierte en materia de importancia fundamental para las comunidades locales y para su vida diaria, al requerir de la participación e incorporaciones de estos puntos de vista en el proceso de toma de decisiones. Las percepciones son muy diversas y dependen de varios aspectos tales como el nivel socioeconómico, las estrategias de subsistema, la afiliación política o el acervo cultural entre otros factores (Gerritsen et al, 2003). El estudio de las percepciones es fundamental ya que permitirá entender los vínculos entre medio ambiente, problemas mundiales y su aplicación a la ingeniería en los estudiantes de Ingeniería Mecatrónica de la Universidad Politécnica de Sinaloa.

\section{RECOPILACIÓN DE DATOS}

El instrumento consistió en un cuestionario compuesto de 31 preguntas y afirmaciones de las variables a medir. El instrumento es una adaptación del cuestionario utilizado por el Grup de Recerca Educacio i Ciutadani (GREIC) de la Universitat de les Illes Belears, que surgió de la investigación: Training European Teachers for Sustentable Development and Intercultural Sensivit. (Oliver, 2005). Las preguntas fueron organizadas en cinco grupos. El primero identifica aspectos sobre ecología y medio ambiente, el segundo las causas de los problemas ambientales y la disponibilidad que tiene para invertir su dinero. En el tercer grupo permiten identificar la concepción que tienen del desarrollo económico y ambiente, mientras que el cuarto grupo identificar la importancia que tienen la ciencia y la tecnología en relación con el ambiente y el quinto grupo tenía el propósito de analizar el desarrollo profesional de un Ingeniero en Mecatrónica.

Para la estimación de la confiabilidad del instrumento, se aplicó la medida de consistencia interna denominada alfa de Cronbach ( $\alpha$ ). El instrumento presentó un coeficiente de 0.78 , lo que representó una confiabilidad aceptable para el instrumento; toda estadística manejada se obtuvo mediante la utilización del paquete estadístico para ciencias sociales (SPSS v17). Para esta investigación se limitó solamente a la Universidad Politécnica de Sinaloa, México; como la población o universo. El universo de investigación estuvo integrado por estudiantes activos a nivel licenciatura de la carrera Ingeniería Mecatrónica que oferta la Universidad Politécnica de Sinaloa, en el turno matutino, correspondiente al ciclo escolar 2011-2012, con una matrícula de 116 estudiantes (Upsin, 2012). Se calculó un tamaño muestra de 80 encuestas con un margen de error máximo permitido, estimado en $6 \%$ y con un nivel de confianza del 0.95 de probabilidad de que los resultados obtenidos fueran válidos. La fórmula para realizar el cálculo anterior es la siguiente:

$$
n=\frac{z^{2}[N(p)(d)]}{e^{2}(N-1)+z^{2}(p)(q)}
$$

En esta ecuación:

$\mathrm{n}=$ Tamaño de la muestra requerida.

z= Nivel de Confiabilidad (95\% valor estándar de 1.96, según tabla del área bajo la curva normal).

$\mathrm{N}=$ Tamaño de la población (116 estudiantes de Ingeniería Mecatrónica).

$\mathrm{p}=$ Variabilidad positiva $(0.5)$.

$\mathrm{q}=$ Variabilidad negativa $(0.5)$.

$\mathrm{e}=$ Margen de error permitido $(6 \%)$. 


\section{ANÁLISIS DE DATOS Y RESULTADOS}

Se describen los datos obtenidos durante análisis descriptivo consistente en el empleo de medidas de tendencia central y de frecuencias para clasificar los datos acerca del tema de trabajo y determinar el alcance de los objetivos planteados al inicio de este proceso. El estudio ha sido clasificado como un diseño no experimental, transversal y descriptivo. No experimental porque no se pueden manipular las variables, los datos a reunir se obtendrán de los estudiantes, transversal ya que la recolección de datos se realiza en un solo tiempo. La población estuvo conformada por 80 estudiantes de la carrera de Ingeniería Mecatrónica, de los cuales 15 eran mujeres (19\%) y 65 hombres (81\%). La edad de los estudiantes oscilo entre los 18 y 27 años. De acuerdo con el cuatrimestre que se cursaba, estuvo distribuido de la siguiente manera: de "Noveno" representaban el (36\%); de "Séptimo" (46\%); y "Cuarto" (14\%). Como ya se comentó, las preguntas fueron organizadas en cinco grupos; el primero identificaba las ideas que se tiene respecto a ecología y medio ambiente. En la pregunta $1 \mathrm{se}$, menciona lo siguiente: Cuando hablamos de naturaleza, ¿Qué significa para ti? Se encontró que en el caso del término "naturaleza", la opción a la respuesta "concepto" alcanzó el (35\%) de la población, lo cual parece indicar que la población tiene claro el significado de naturaleza: el (30\%) de la población lo relacionó con "plantas", seguido con "animales" con un $(25 \%)$. La respuesta con menor frecuencia fue problemas relacionados, con un (10\%).La respuesta "no sé" no tuvo resultados. En la figura 1, se observan los resultados relacionados con la pregunta Cuando hablamos de naturaleza, ¿Qué significa para ti?

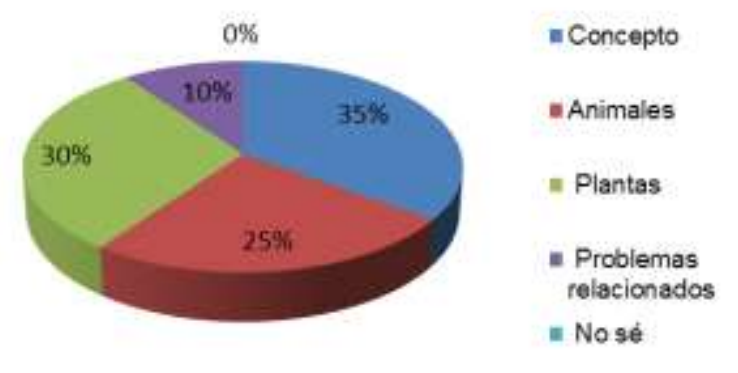

Fig. 1. Significado de naturaleza para los estudiantes de Ingeniería Mecatrónica.

Con respecto a la pregunta 2, Cuando hablamos de medio ambiente, ¿Qué significa para ti?, al realizar el análisis se encontró lo siguiente. La respuesta "problemas relacionados" presentó la mayor frecuencia con el $(41 \%)$ de la población. La respuesta "concepto" fue la segunda elección con el (34\%); seguido por "plantas" con el (16\%); y "animales" con el (9\%); la opción "no sé" no obtuvo resultados. (Fig.2).

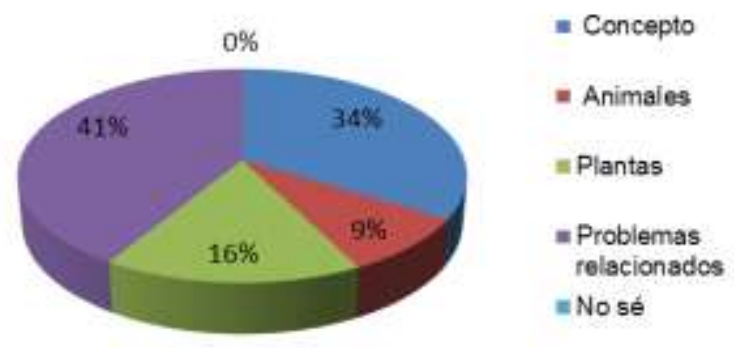

Fig. 2. Significado del medio ambiente de los estudiantes de Ingeniería Mecatrónica.

Los estudiantes de Ingeniería Mecatrónica, al considerar la relación con la naturaleza y medio ambiente, señalan que "la especie humana es una más del planeta y debería mantener una relación de interdependencia con las demás especies" (56\%); y "los cambios en el medio ambiente producidos exclusivamente en beneficio personal causan graves problemas" (51\%). Estos resultados parecen mostrar que los estudiantes tienen una actitud respetuosa con el medio ambiente. Con base a la problemática ambiental generada por el ser humano, se cuestionó a los estudiantes: ¿Cuáles son los problemas ambientales que más afectan a tu vida? En la figura 3, se muestran los resultados; el (43\%) de la población considera que la "contaminación del agua" afecta en gran medida su calidad de vida; seguida por la "generación de residuos con un (39\%) y por último, la destrucción de áreas verdes con un (18\%). 


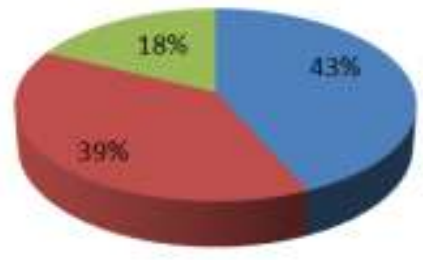

\author{
ะ1. Contaminación del \\ agua \\ *2. Generación de \\ residuos \\ *3. Destrucción de \\ arreas verdes
}

Fig. 3. Percepción de los estudiantes de la problemática ambiental ocasionada por el ser humano.

Los estudiantes mostraron tener conocimiento sobre las problemáticas ambientales, por lo que se cuestionó: ¿Qué tanto sabe sobre los siguientes temas?, a partir de los resultados se mencionan aquellos problemas en que los estudiantes señalan tener más conocimiento y aquellas que desconocen más, para el análisis del conocimiento sobre problemas ambientales, se considera únicamente la respuesta "conozco el tema a fondo" y "sé algunas cosas sobre el tema".

El reciclaje, los cambios climáticos, la contaminación del agua potable, la contaminación atmosférica, el agujero de la capa de ozono, (representan el 8\%). Deforestación, aprovechamiento del agua, lluvia ácida (representan el 7\%) (Por orden de mayor a menor conocimiento). La Planificación Local, Desertificación, Residuos nucleares, (representan el 3\%); Comida manipulada genéticamente, Planificación urbana (representan el $3 \%$ ); la valoración del impacto ambiental, tratamiento de residuos (representan el $5 \%$ ) (ordenadas de menor a mayor conocimiento). En la figura 4, se observa los resultados relacionados con la pregunta ¿Qué tanto sabe sobre los siguientes temas?

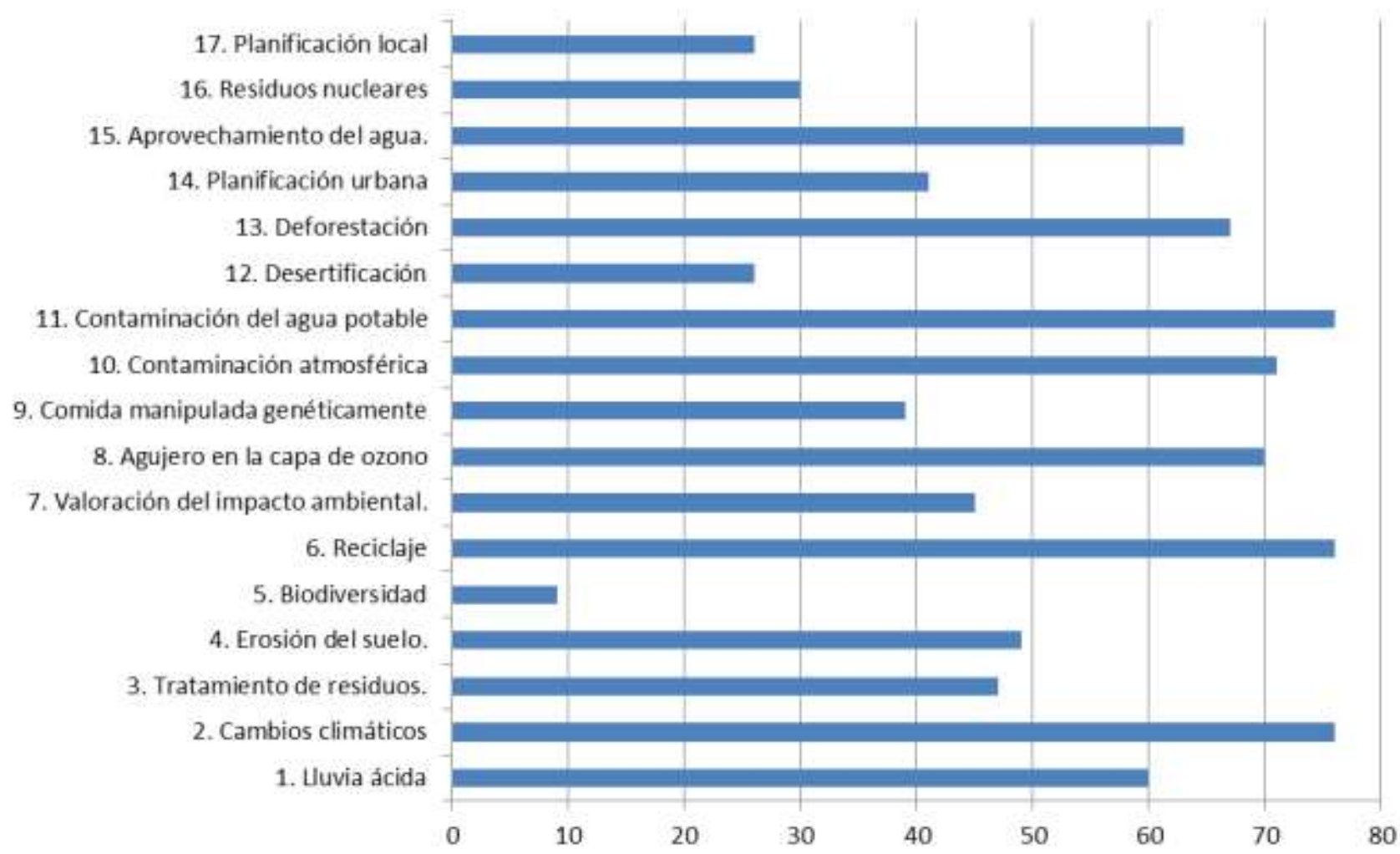

Fig. 4. Muestra el conocimiento sobre temas relacionados con la problemática ambiental.

La segunda etapa de la encuesta atendió la preocupación sobre el medio ambiente. Los estudiantes manifestaron una mayor preocupación por el estado del medio ambiente a nivel "en el mundo" con un (42\%); con respecto lo que sucede "en tu país" (30\%); "en tu región" (14\%); y "en tu localidad" (14\%). En la figura $5 \mathrm{se}$, incluyen los resultados de la pregunta ¿Grado de preocupación sobre el medio ambiente en relación a tu entorno?; Se consideró únicamente la respuesta me preocupa mucho. 


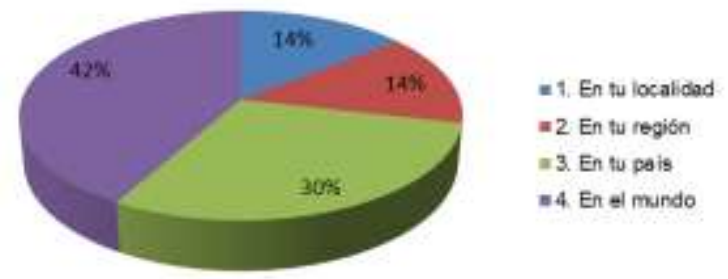

Fig. 5. Aspectos de la situación, actual, a nivel local, regional y global desde el punto de vista de los estudiantes de Ingeniería Mecatrónica.

Para analizar el grado de inquietud por los problemas ambientales en el país, se formuló la pregunta: ¿Cómo crees que son los siguientes problemas ambientales?. Para el análisis de esta pregunta se considera únicamente la respuesta de la columna "muy grave". La problemática ambiental con mayor porcentaje es la relacionada con los "Residuos industriales" (59\%); le siguen, "Poco interés en la conservación de la naturaleza y las especies" (58\%); "Contaminación ambiental" (55\%); "Contaminación de mares y playas" (55\%); "Contaminación de ríos, lagos y embalses" (50\%). En la Tabla 1 se presenta los resultados a la pregunta ¿Cómo crees que son los siguientes problemas ambientales?

Tabla 1. Pensando en tu país, ¿Cómo crees que son los siguientes problemas ambientales?

\begin{tabular}{|l|l|l|l|l|l|}
\hline & Nada grave & Un poco grave & Grave & Muy grave & No sé \\
\hline 1. Ruido. & $(10 \%)$ & $(41 \%)$ & $(38 \%)$ & $(6 \%)$ & $(5 \%)$ \\
\hline $\begin{array}{l}\text { 2. Uso de pesticidas y productos químicos en la } \\
\text { agricultura. }\end{array}$ & $(1 \%)$ & $(35 \%)$ & $(39 \%)$ & $(22 \%)$ & $(3 \%)$ \\
\hline 3. Descenso de la población en algunas áreas. & $(19 \%)$ & $(49 \%)$ & $(12 \%)$ & $(6 \%)$ & $(14 \%)$ \\
\hline 4. Calidad del agua potable. & $(1 \%)$ & $(14 \%)$ & $(40 \%)$ & $(45 \%)$ & $(0 \%)$ \\
\hline 5. Contaminación ambiental. & $(0 \%)$ & $(5 \%)$ & $(40 \%)$ & $(55 \%)$ & $(0 \%)$ \\
\hline 6. Contaminación de mares y playas. & $(0 \%)$ & $(9 \%)$ & $(36 \%)$ & $(55 \%)$ & $(0 \%)$ \\
\hline 7. Incendios forestales. & $(2 \%)$ & $(28 \%)$ & $(44 \%)$ & $(25 \%)$ & $(1 \%)$ \\
\hline 8. Residuos urbanos. & $(0 \%)$ & $(7 \%)$ & $(50 \%)$ & $(40 \%)$ & $(3 \%)$ \\
\hline 9. Residuos industriales. & $(0 \%)$ & $(12 \%)$ & $(26 \%)$ & $(59 \%)$ & $(3 \%)$ \\
\hline 10. Contaminación de ríos, lagos y embalses. & $(0 \%)$ & $(13 \%)$ & $(36 \%)$ & $(50 \%)$ & $(1 \%)$ \\
\hline 11. Despilfarro de energía. & $(0 \%)$ & $(19 \%)$ & $(31 \%)$ & $(45 \%)$ & $(5 \%)$ \\
\hline 12. Aguas residuales sin tratar. & $(3 \%)$ & $(23 \%)$ & $(41 \%)$ & $(28 \%)$ & $(5 \%)$ \\
\hline 13. Tráfico. & $(6 \%)$ & $(29 \%)$ & $(45 \%)$ & $(19 \%)$ & $(1 \%)$ \\
\hline $\begin{array}{l}\text { 14. Poco interés en la conservación de la naturaleza } \\
\text { y las especies. }\end{array}$ & $(1 \%)$ & $(7 \%)$ & $(34 \%)$ & $(58 \%)$ & $(0 \%)$ \\
\hline 15. Incremento de urbanización ilegal. & $(4 \%)$ & $(28 \%)$ & $(44 \%)$ & $(14 \%)$ & $(9 \%)$ \\
\hline 16. Destrucción de lugares históricos. & $(10 \%)$ & $(37 \%)$ & $(34 \%)$ & $(18 \%)$ & $(1 \%)$ \\
\hline
\end{tabular}

A los encuestados se les preguntó también ¿Cuáles crees que son las causas principales de la contaminación del agua? La principal respuesta de la contaminación del agua, fue "Residuos industriales" (36\%). El segundo lugar lo ocupó la "Falta de sanciones y multas para quienes contaminan" (27\%). El "Uso de pesticidas" (8\%); la "Falta de acción legal" (11\%) y "Mala calidad del sistema de cloacas" (17\%); son consideradas las causas menos probables de contaminación del agua por parte de los estudiantes. La opción "Sequía" no obtuvo resultados. A continuación se les solicitó que respondieran al siguiente cuestionamiento ¿Cuáles crees que son las causas principales de los problemas de residuos? y, se encontró que los principales problemas de residuos son: la "Falta de cooperación activa de los ciudadanos" (23\%), seguido de la "Falta de respeto de la gente" (22\%); la "Falta de responsabilidad de las empresas" (19\%); la "Falta de planificación nacional y municipal para el tratamiento de residuos" (18\%); y "Falta de actuación de los Ayuntamientos" (11\%). Referente a la pregunta: ¿Cuáles crees que son las causas principales del deterioro de los lugares de patrimonio histórico?, los encuestados coincidieron en señalar que la "Falta de interés por parte de la gente" (36\%); y la "Falta de interés por parte de las autoridades" (27\%); eran las principales causas del deterioro del patrimonio histórico. 
En respuesta a la interrogante, ¿En qué fuentes de energía debería invertir México?, los estudiantes mostraron una marcada tendencia hacia la inversión en energías renovables especialmente con la "Solar" (38\%); y la "Eólica" (22\%). Como inversiones prioritarias, le siguen: la "Hidroeléctrica" (14\%); y el "Petróleo" (13\%). Las de menor prioridad fueron "Mareomotriz" (9\%); "Nuclear" (2\%); "Carbón" (1\%); y "Gas" (1\%). Se puede observar que el porcentaje de energías renovables (Solar, Eólica y Mareomotriz) representa el 69\% frente al porcentaje de las energías convencionales (Carbón, Petróleo, Madera, Turba, Nuclear, Hidroeléctrica y Gas) con un 31\%. Se propuso a los estudiantes analizar el grado de implicación personal en la protección del medio ambiente, ¿Te parecería bien pagar...? el (65\%) estaría dispuesto a pagar más dinero si "Hubiera marcas de productos que fueran respetuosos con el medio ambiente" y "Más impuestos si supieras que el dinero se emplearía para proteger el medio ambiente" $(50 \%)$ y por último "Precios más altos para que las empresas comerciales pudieran preservar mejor el medio ambiente" con un (28\%).

El patrimonio histórico es una parte importante de cada municipio, por lo que se cuestionó a los estudiantes acerca de la intervención municipal respecto a la conservación de tal patrimonio. La opción con mayor porcentaje fue: "El Ayuntamiento debería trasladar el monumento a otro lugar" (33\%). La segunda opción fue "El Ayuntamiento sólo debería permitir la circulación de transporte público" (32\%), seguida de "EI Ayuntamiento debería restaurar el monumento" (25\%). Entre las últimas opciones se encuentra "EI Ayuntamiento debería introducir estacionamientos de pago en la zona" (8\%) y "El Ayuntamiento no debería permitir la circulación de vehículos" (2\%).

Referente a la actuación mundial para un mundo mejor, se les solicitó a los estudiantes que seleccionaran las consideraciones más importantes con respecto al "Cambio climático / Calentamiento global". Respecto a la relación que debe existir entre el crecimiento económico y protección del medio ambiente, se puso de manifiesto la "Necesidad de garantizar el crecimiento económico respetando también la protección del medio ambiente" (92\%) seguida por la "La protección del medio ambiente debería ser más importante que el crecimiento económico" (8\%) (Fig. 6).

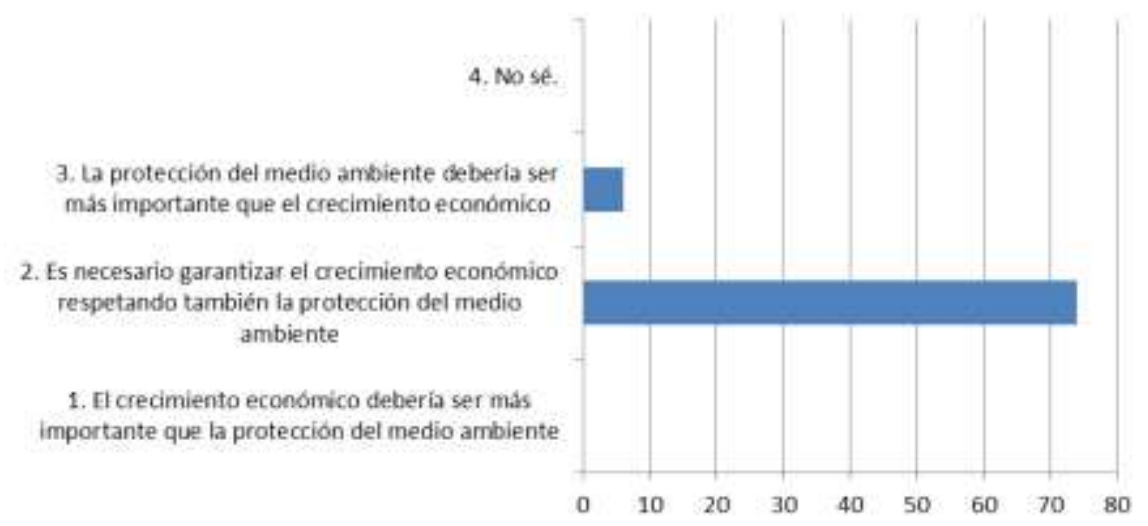

Fig. 6. Aspectos importantes que consideran los estudiantes para mejorar el cambio climático/calentamiento global.

Se cuestionó a los estudiantes sobre la relación entre medio ambiente, ciencia y tecnología. Un porcentaje elevado se inclinó por "La ciencia y la tecnología como causa y solución de los problemas ambientales" (79\%); seguida por: "Los avances científicos y tecnológicos solucionarán los problemas ambientales dentro de 10/15 años" (19\%); las otras opciones se mantienen entre el (1\%) y (4\%). Se preguntó a los estudiantes si estaban de acuerdo con determinadas propuestas para la solución de los problemas ambientales relacionadas con la participación ciudadana. Para este análisis se seleccionaron únicamente las respuestas afirmativas. En la Tabla 2, se muestran los resultados, que incluyen. "La participación de los ciudadanos será necesaria para solucionar los problemas ambientales" (53\%), la "Necesidad de que la gente cambie los hábitos de consumo" (45\%); y "No tiene sentido hacer algo ya que los problemas no tienen solución" (2\%).

Tabla 2. En relación con la solución de problemas ambientales, indica si estás de acuerdo con las siguientes afirmaciones.

\begin{tabular}{|l|c|c|c|c|}
\hline & No & En parte & Sí & No sé \\
\hline $\begin{array}{l}\text { 1. La participación de los ciudadanos será necesaria para } \\
\text { solucionar los problemas ambientales. }\end{array}$ & 0 & 5 & 74 & 0 \\
\hline 2. Será necesario que la gente cambie sus hábitos de consumo. & 0 & 16 & 64 & 0 \\
\hline $\begin{array}{l}\text { 3. No tiene sentido intentar hacer algo porque los problemas } \\
\text { ambientales graves no tienen solución. }\end{array}$ & 68 & 7 & 3 & 1 \\
\hline
\end{tabular}


Se preguntó a los estudiantes si conocían organizaciones que trabajan por un futuro mejor, a nivel local, nacional e internacional. Las cinco organizaciones más conocidas por los estudiantes son: "Green Peace" (30\%); "ONU" (20\%); "UNICEF" (9\%); "UNESCO" (7\%); "OMS" (4\%).Se solicitó a los estudiantes, que indicasen, su grado de confianza en las organizaciones/personas para resolver los problemas del medio ambiente. Los estudiantes mostraron su confianza a los "Ciudadanos" (26\%); el "Gobierno" (17\%); "Tú" (16\%); y las Organizaciones internacionales (Las Naciones Unidas, Green Peace, etc.) (12\%). Sin embargo, existe poco nivel de confianza en los "Periódicos" (10\%); las "Escuelas" (4\%); los "Ayuntamientos" (4\%); "Científicos" (3\%); y "Gente famosa" (1\%). Referente a la actuación personal para un medio ambiente mejor, se les preguntó ¿Cuál de estas acciones has realizado o te gustaría realizar para hacer que el mundo sea un sitio mejor? Los estudiantes manifestaron: "Utilizar bombillas de bajo consumo" (16\%), seguida, "Ahorrar energía en casa" (14\%), "Intentar usar menos agua" (13\%), y "Usar papel reciclado" (10\%). Las acciones menos realizadas fueron: "Evitar usar el coche para trayectos diarios" (9\%), "Participar en actividades que mejoren el medio ambiente" (7\%), y "Escoger productos con envoltorios reciclables" (7\%). Se preguntó a los estudiantes cual sería el principal reto de Ingeniería Mecatrónica en el futuro. Mencionaron que la "Energía y medio ambiente" (22\%); la "Robótica" (16\%), y el "Desarrollo sustentable" (11\%). Las menos importantes fueron: el "Cambio climático" (5\%), "Materiales" (5\%), "Educación" (4\%), "Suministro y administración de agua" (3\%), y por último: "Medio ambiente" (1\%). En la Tabla 3, se observan los resultados a la pregunta ¿Cuál crees que sea el principal reto de la ingeniería Mecatrónica en el futuro?

Tabla 3. ¿Cuál crees que sea el principal reto de la ingeniería Mecatrónica en el futuro? Escoge las tres que consideres más importantes.

\begin{tabular}{|l|c|c|}
\hline 1. Cambio climático. & 12 & $5 \%$ \\
\hline 2. Energía y medio ambiente. & 53 & $22 \%$ \\
\hline 3. Desarrollo sustentable. & 27 & $11 \%$ \\
\hline 4. Suministro y administración del agua. & 8 & $3 \%$ \\
\hline 5. Agricultura y medio ambiente. & 3 & $1 \%$ \\
\hline 6. Reusó y reciclamiento y medio ambiente. & 20 & $9 \%$ \\
\hline 7. Transporte público y medio ambiente. & 10 & $4 \%$ \\
\hline 8. Materiales. & 12 & $5 \%$ \\
\hline 9. Comunicaciones. & 7 & $3 \%$ \\
\hline 10. Robótica. & 39 & $16 \%$ \\
\hline 11. Ingeniería Biomédica. & 21 & $9 \%$ \\
\hline 12. Seguridad. & 18 & $8 \%$ \\
\hline 13. Educación. & 9 & $4 \%$ \\
\hline
\end{tabular}

Ante la pregunta ¿Cómo un profesional de la Ingeniería Mecatrónica puede incorporar en sus actividades a los planteamientos del Desarrollo Sustentable?, los estudiantes opinaron que con el "Desarrollo de nuevas tecnologías para cumplir con los grandes retos en energía, medio ambiente, alimentación, vivienda, agua, transporte, seguridad y salud" (43\%), "La Asociación de los profesionales con el placer (compromiso) de descubrir, crear y aplicar las soluciones de ingeniería para mejorar la vida del ser humano" (34\%), "Creación de soluciones globales y sustentables de ingeniería que satisfagan las necesidades básicas de toda la gente" (22\%), y por último, "Fomento a la asociación global y al desarrollo local apropiado" (1\%). Se cuestionó a los participantes si deberían asumir mayor responsabilidad hacia un futuro sustentable. La opinión mayoritaria fue "Siempre" con un (60\%) y le siguieron "Casi siempre" (30\%), y finalmente, "Algunas veces" (10\%). Las opciones "Rara vez" y "Nunca" no obtuvieron simpatizantes.

Tomando en cuenta los aspectos ambientales que debe integrar un profesional en Ingeniería Mecatrónica se preguntó: ¿Qué aspectos ambientales debe integrar como objetivos sostenibles en sus actividades profesionales un Ingeniero Mecatrónico? Recabamos las siguientes opiniones: "Identificar, evaluar y seleccionar la mejor solución en el proyecto" (26\%), "Minimizar el consumo de recursos" (21\%), "Partir del conocimiento del entorno" (14\%). Las respuestas con menores frecuencias fueron: "Respetar la legislación ambiental" (12\%), "Integrar el trabajo en equipo, multi e interdisciplinar" (8\%), "Los costos ambientales" (7\%), "Minimizar la emisión" (7\%), y por último: "Procurar los ciclos cerrados en el uso de los recursos" (12\%). Se preguntó a los encuestados: ¿Cuáles deberán ser las medidas mínimas de control a tomar en cuenta en la ejecución de una obra de ingeniería?, obteniendo las siguientes aseveraciones: "Protección de la vegetación, de los recursos hídricos, sistemas medios marinos" (28\%), "Protección de la fauna" (22\%), "Protección atmosférica y acústica" (19\%), y "Protección de los suelos" (14\%). Las repuestas con menores porcentaje fueron: "Protección del patrimonio cultural" (9\%); y "Protección del paisaje" (8\%). Por último se preguntó: ¿Consideras importante que se incluyan asignaturas de desarrollo sustentable y educación 
ambiental en tu plan de estudios?. Los resultados revelan que con un (50\%); están "Totalmente de acuerdo" que se incluyan la asignatura de desarrollo sustentable y educación ambiental en su plan de estudio, le siguen: "De acuerdo" con (38\%), "Ni en acuerdo, ni en desacuerdo" (11\%). Solo el (1\%), está "En desacuerdo" y nadie respondió "Totalmente en desacuerdo" $(0 \%)$.

Con base a los resultados obtenidos en este estudio se puede observar que se requiere mejorar el conocimiento ambiental de los estudiantes de Ingeniería Mecatrónica, proporcionando una formación que incluya aspectos actuales, a nivel local, regional y global. Parece existir un vínculo entre las actitudes ambientales de los encuestados y la concepción de naturaleza y medio ambiente. Las mayorías de los estudiantes tienen la capacidad de identificar la problemática ambiental actual. Así mismo, parecen comprender los daños que ocasiona la contaminación en general. Como también reconocen que el ser humano es el origen de estos fenómenos. Se pone de manifiesto la necesidad de garantizar el crecimiento económico respetando también la protección del medio ambiente. Con relación a la Ciencia y la Tecnología, donde un $79 \%$ de los estudiantes consideraron que son la causa y la solución a los problemas del medio ambiente. Es aquí donde la Ciencia y la Tecnología, deben tener un papel decisivo en la búsqueda de alternativas para lograr un verdadero desarrollo sustentable y que se implementen planes de acción que cubran las siguientes áreas: Eficiencia energética, Usos de energías renovables y Tecnologías verdes. La Ingeniería Mecatrónica debe desarrollar nuevas tecnologías y técnicas que apoyen el crecimiento y promuevan el desarrollo sustentable, para cumplir con los retos en energía, medio ambiente, alimentación, vivienda, agua, transporte, seguridad y salud.

\section{CONCLUSIONES}

Después de conocer la percepción que tiene los estudiantes de Ingeniería Mecatrónica de la Universidad Politécnica de Sinaloa, México; con respecto al problema ambiental y dar respuestas a la pregunta de investigación planteada, así como del análisis de la información, se concluye lo siguiente: El estudio pone en evidencia la importancia que los estudiantes le otorgan al medio ambiente para disfrutar de un mundo mejor. La contaminación y destrucción de los recursos naturales son percibidos como los principales problemas del entorno en el que viven. La ciencia y la tecnología son consideraros como la causa y la solución de los problemas del medio ambiente. El estudio sugiere la necesidad de incluir en los planes de estudio de la carrera de Ingeniería Mecatrónica la materia de educación ambiental. Propiciar en los estudiantes diversos enfoques del desarrollo sustentable, tanto sociales, económicos, culturales y políticos de los problemas ambientales y fomentar los valores que requiere un Ingeniero Mecatrónico en su profesión y en particular el de poseer una actitud comprometida con el cuidado del medio ambiente.

\section{REFERENCIAS}

Aragonés, J.I., V. Sevillano, B. Cortés y M. Amérigo, Cuestiones Ambientales que se perciben como problemas, Medio ambiente y comportamiento Humano Rev. Internacional de Psicología Ambiental 7, (2), 119, (2006).

Barraza, L. y Ceja, M.P., Los niños de la comunidad: su conocimiento y su percepción de la naturaleza (2003), http://www2.inecc.gob.mx/publicaciones/libros/420/dieciseis.html Acceso: 25 de Noviembre (2014).

CAETS, Consejo de academias de ingeniería. Programa estratégico estudio del estado del arte y prospectivas de la ingeniería en México y el mundo (2009) http://www.ai.org.mx/ai/images/sitio/edodelarte /2010/10.prospectiva_de_la_ingenieria_en_mexico_y_en_el_mundo.pdf. Acceso: 30 de mayo (2012).

Castells, M. y M. Morey, La percepción de los problemas ambientales y la visión del futuro en los jóvenes españoles. Actas del VII Congreso Nacional de Medio Ambiente, 1-24, Madrid, España, 26 a 20 de Noviembre (2012).

Cerrillo, J.A., Medición de la conciencia ambiental: Una revisión crítica de la obra de Riley. DOI: http://dx.doi.org/10.5565/rev/athenead/v0n17.609, Rev. Athenea Digital (en línea) (17), 33-52, (2010).

Echeverría J., Reflexiones sobre el futuro de la ingeniería en México. Academia de Ingeniería (2011), http://www.ai.org.mx/archivos/coloquios/1/Reflexion_sobre_el_Futuro_de_la_Ingenieria/Reflexion\%20sobre \%20el\%20Futuro\%20de\%20la\%20Ingenieria_escrito.pdf. Acceseso: $10 \overline{\text { de }}$ marzo (2011).

Espejel a., A. Flores, Educación ambiental escolar y comunitaria en el nivel medio superior, Revista Mexicana de investigación educativa, 17, (55), 1173-1199 (2012). 
Espino, P., A. Lizárraga, C. F. Montoya y V. M. Rodríguez Velázquez, La Ingeniería Mecatrónica y su Contribución al Desarrollo Sustentable. Memoria del 10 Congreso Nacional de Mecatrónica, 1-5, Puerto Vallarta, Jalisco, México, 3 y 4 Noviembre (2011).

Gerritsen, P.W., M. Montero, P. Figueroa, El mundo en un espejo. Percepciones campesinas de los cambios ambientales en el Occidente de México. Revista Economía, Sociedad y Territorio, 4, (14), 253-278 (2003).

Márquez, R.I., O.O. Salavarría, A. Eastmond, M. E. Ayala, M.A. Arteaga, A. P. Isaac-Márquez, J.L. Sandoval, y L.A. Manzanero, Cultura ambiental en estudiantes de bachillerato: Estudio de caso de la educación ambiental en el nivel medio superior de Campeche. Revista electrónica de investigación educativa, 13(2), 83-99. (2011).

Nellemann, C., E. Corcoran. Dead Planet, Living Planet - Biodiversity and Ecosystem Restoration for Sustainable Development (2010), http://www.unep.org/pdf/RRAecosystems_screen.pdf. Acceso: 28 de Enero (2014).

Oliver, M., M. Castells, A. Casero y M. Morey. Actitudes y percepción del medio ambiente en la juventud española (2011), http://www.marm.es/es/ceneam/recursos/documentos/serieea/actitudes.aspx. Acceso: 10 de mayo (2011).

Rodríguez, M., Ingeniería y medio ambiente, Revista de Ingeniería, (26), 56-63 (2007).

Padilla, L. S., A.M. Luna, Percepción y conocimiento Ambiental en la costa de Quintana Roo: una caracterización a través de encuestas, Revista Investigaciones Geográficas, Boletín del Instituto de Geografía, (52), 99-116 (2003).

Sosa, S.B., R.I. Márquez, R, A. Eastmond, M.E. Ayala y M.A. Arteaga, (2010). Educación superior y cultura ambiental en el sureste de México, Universidad y ciencia, 26(1), 33-49 (2010).

UNESCO, Ciencia y Tecnología para el Desarrollo. Oficina de información pública (2006), http://www.unesco.org/bpi/pdf/memobpi20_science_es.pdf. Acceso: 4 de Junio (2011).

UMAI, Unión Mexicana de Asociación de Ingenieros. Foro Panamericano sobre Contribuciones de la Ingeniería al Mejoramiento del Medio Ambiente (2009), http://www.umai.org.mx/Noticias/2009/Foro_API/doc_spa.pdf. Acceso: 5 de Abril (2011).

UPSIN, Universidad Politécnica de Sinaloa. Estadísticas, Ciclo escolar 2011-2012 (2012) http://www.upsin.edu.mx/images/normatividad/estadistica_ciclo_escolar_2010-11.pdf . Acceso: 5 de Abril (2012).

Zamorano, B., V. Parra, F. Peña, J.L. Vargas y Y. Castillo, Compromiso ambiental de los estudiantes del nivel Medio superior, Revista Desarrollo Local Sostenible, Red Académica Iberoamericana Local Global, 4, (11), 1-13 (2011). 